\title{
Low-Cost Drugs Still Relevant in the Present-Day Era
}

\author{
Subhashchandra Daga
}

\section{ABSTRACT}

Escalating medical costs contribute to poverty in countries with low resources. The drug costs account for 17 percent of medical expenses. Revisiting time-tested, cost-effective drugs can reduce these costs. Some of them find a place in the WHO Model List of Essential Medicines for Children. The list consists of medicines for a basic healthcare system. They are safe and cost-effective. The present paper identifies co-trimoxazole and chloramphenicol as antimicrobials, chloroquine for malaria, adrenaline, theophylline for asthma, and phenobarbital as an anti-epileptic drug that merits consideration for reviving interest in them and reduce drug treatment costs.

What is already known about this subject?

- The cost of drugs contributes to rising medical costs.

- Medical expenses push a large population below the poverty line.

What does this study add?

- Rediscovering the relevance of old low-cost drugs is essential.

- Revisiting the WHO Model List of Essential Medicines for Children may be useful.

- Drugs such as chloramphenicol and theophylline are such examples.

Keywords: Low-cost drugs, Medical costs, medical expenses, medical treatment and poverty, WHO essential drugs list.

\author{
Published Online: December 3, 2021 \\ ISSN: $2736-5476$ \\ DOI: $10.24018 /$ ejclinicmed.2021.2.6.134 \\ S. Daga* \\ Emeritus Prof. B.J. Medical College and \\ Sassoon General Hospital, India. \\ (e-mail: dagasubhash49@gmail.com) \\ *Corresponding Author
}

\section{INTRODUCTION}

The expenses associated with prescription drugs are rising and account for 17 percent of the overall medical costs [1]. Over 800 million people spend at least 10 percent of their household budgets to pay for health care [1]. This expense pushes around 100 million into extreme poverty [2]. Less than 15 percent of the population in India has a healthcare cover [3]. New drugs displace old drugs, not necessarily for consideration of effectiveness. Many times, the use of newer drugs and the side-effects of older drugs are amplified. Coupled with this, lack of commercial interest or commercial neglect facilitates a cheap drug to fade away, which hits lowresource countries hard. Rediscovering the relevance of old drugs in present times for their cost-effectiveness may reduce medical costs.

The present study highlights the cost-effectiveness of some of the drugs that merit consideration. Based on which, cotrimoxazole and chloramphenicol as antimicrobials, chloroquine for malaria, adrenaline, and theophylline for asthma, and phenobarbital as anti-epileptic drugs from the WHO Model List of Essential Medicines for Children [4] may be reconsidered for use. These drugs are prescribed for a basic healthcare. They are safe and cost-effective medicines [4].

\section{Cotrimoxazole For FeVer}

Cotrimoxazole is among the key access essential drugs [4]. It is effective against $S$. pneumoniae and $H$. influenzae that forms the pneumonia case management strategy of the World
Health Organization (WHO) [5]. These organisms also cause occult bacteremia, a significant cause of fever in children [6]. S. pneumoniae caused the majority $(80 \%)$ of occult bacteremia before routine immunization. $\mathrm{H}$. influenzae type $\mathrm{b}$ caused bacteremia in a smaller percentage $(10 \%)$ and an even smaller percentage $(5 \%)$ by N. meningitides. Cotrimoxazole is also a potent drug against $\mathrm{E}$. coli that cause urinary tract infection, another important cause of fever [7]. Coagulase-negative staphylococci cause bacteremia in malnourished children [8], and co-trimoxazole is a good antistaphylococcal drug [9]. In a study, co-trimoxazole susceptibility increased progressively from $31 \%$ in 1988 to $92 \%$ in $1997 \quad(\mathrm{p}<0.0001)$. It is inexpensive, is orally administered, and has a convenient twice-a-day schedule. A study on syndromic management of fever used cotrimoxazole and chloroquine as the first-line treatment in stable children with fever [10]. Of the 81 children, 71 (87.3\%) responded.

At present, the WHO recommends amoxicillin in place of co-trimoxazole for pneumonia treatment [11]. The new classification includes two categories of pneumonia; pneumonia with fast breathing with or without chest indrawing, which requires home therapy with oral amoxicillin, and "severe pneumonia," pneumonia with any general danger sign requires a referral and injectable therapy. The cost of 5 days of treatment ( $20 \mathrm{~kg}$ child) for Amoxycillin \& co-trimoxazole is approximately 0.8 and 0.25 USD, respectively. 


\section{Chloramphenicol fOR PNEUMONIA}

Chloramphenicol, another key drug, has been recommended by the WHO for severe pneumonia [5]. Currently, the WHO recommends parenteral administration of penicillin and gentamicin and facility-based care for severe pneumonia [11]. Most importantly, chloramphenicol is effective parenterally and orally, with a bioavailability of $80 \%$ [12]. As a result, a step down from parenteral administration during the acute phase to oral administration during the recovery phase is possible without compromising efficacy. The home treatment is possible during the recovery stage with oral medication if the parents insist upon an early discharge. In a study comprising of 5464 patients, 170 patients, representing 3.1\%, were discharged against medical advice (DAMA) [13]. Of these, DAMA was sought in $15.7 \%$ of cases of pneumonia. Lost days at work and the inability to care for other children were important reasons [13]. Oral chloramphenicol allows home treatment and may, therefore, reduce hospital stay DAMA risk, and incomplete treatment. However, chloramphenicol is known to cause both dosedependent and dose-independent blood dyscrasias. The condition is rare, occurring about once in every $18000-50$ 000 subjects in various countries [14]. The cost of pneumonia treatment for seven days of a $20 \mathrm{~kg}$ child with injectable ampicillin and gentamicin, ceftriaxone, and chloramphenicol is 6,9 , and 5 USD, respectively. However, step down care at home is possible only with chloramphenicol.

\section{Chloroquine For MALARIA}

Plasmodium vivax accounts for approximately half of cases of malaria outside Africa [15]. In general, chloroquine is an effective drug for vivax malaria. Chloroquine resistance is still rare but increasing [16]. Chloroquine was withdrawn in Malawi in 1993 but is now an effective treatment for malaria in that country. In 210 children with uncomplicated malaria randomized to chloroquine or sulfadoxine + pyrimethamine. Only one of those who received chloroquine had treatment failure compared with $71 / 87$ of those who got sulfadoxine + pyrimethamine [17]. Artemisinin-based combination therapies (ACTs) are highly effective against $\mathrm{P}$. falciparum, the most prevalent and lethal malaria parasite affecting humans [18]. There are reports of sensitivity returning in falciparum malaria cases since chloroquine was out of use for more than two decades [19]. The cost of treating malaria with chloroquine and ACT for a $20 \mathrm{~kg}$ child is 0.25 and 1.5 USD, respectively.

\section{Adrenaline for ACUTE Asthma}

A guideline based on the literature review supports nebulized salbutamol as the most appropriate therapy for acute asthma [20]. The aerosol therapy may be challenging in the presence of interrupted supplies of nebulization fluid, unfavorable nurse-patient ratio, and too many patients receiving nebulization on the only machine available, delays in repairs of the nebulization unit after a breakdown, and interrupted power supply. An alternative to aerosol therapy is essential in such situations. Subcutaneous adrenaline may be one option [21], [22]. In a double-blind trial, the efficacy and safety of inhaled salbutamol, nebulized with oxygen by face mask, and subcutaneous epinephrine in 40 children with acute asthma was comparable. In a review article that explored subcutaneous adrenaline's role in managing acute asthma, all the five studies suggested that the bronchodilator effect of subcutaneous adrenaline and inhaled salbutamol was comparable [22]. Reference [23] compared the emergency treatment of severe asthma with conventional methods and with a small dose, $0.3 \mathrm{mg}$ subcutaneous, adrenaline, besides. The adrenaline group had rapid onset action and showed better therapeutic effects. The cost of adrenaline ampoule is USD 0.2, and of salbutamol is USD 0.1. However, salbutamol therapy needs a machine and electrical supply for its administration.

\section{TheOPHYLline FOR CHRONIC AsthMA}

Anti-inflammatory drugs have become the mainstay for asthma prophylaxis. Persistent inflammation is a feature of chronic asthma, and there are inflammatory infiltrates in the airway wall, even in mild asthma [24]. The inhaled steroids are the first-line therapy. In resource-scarce settings, there are two limitations. One is the cost of inhalers, and the second, doubts regarding the correct use of the inhalers [25], [26]. The potential of theophylline in asthma prevention needs proper evaluation in such a situation. Once-a-day dosing and oral administration are best suited for such a patient population. Low dose theophylline attenuates the asthmatic reaction to an inhaled allergen [27]. Secondly, the anti-inflammatory effect of theophylline occurs at serum concentrations below the accepted therapeutic range. Hence, the possibility of doserelated side-effects is also reduced [28].

In the before-and-after study, conducted on 40 consecutive children with doctor-diagnosed asthma [29], the patients received sustained-release theophylline at night $(8.00 \mathrm{pm})$ in a single oral dose, $10 \mathrm{mg} / \mathrm{kg}$, for six months. During the preprophylaxis period, 32 events of emergency attendance or hospitalization occurred against only one event recorded with theophylline prophylaxis. This finding is particularly crucial because emergency services are not up-to-the-mark in developing countries [30]. The cost of asthma prevention with an inhaled steroid was Rs.200 (USD 3) per month as against Rs.24 (USD 0.3) for theophylline prophylaxis. Thus, theophylline is an available option where cost and compliance matter most for preventing chronic asthma in children. The monthly expenses on an inhaled steroid and long-acting theophylline for a $20 \mathrm{~kg}$ child are 5 and $0.5 \mathrm{USD}$, respectively.

\section{Phenobarbital for EPILEPSy}

Of the 35 million people with epilepsy who live in developing countries, around $85 \%$ receive no treatment at all [31]. The World Health Organization recommends phenobarbital as the treatment of choice for partial and tonicclonic seizures in resource-restricted countries. Phenobarbital has the following advantages: affordability, broad-spectrum action, absence of major side-effects, linear pharmacokinetics, and once-daily dosing [32]. Banu and colleagues observed comparable seizure control with 
phenobarbital and carbamazepine [33]. They did not observe a significant difference in psychological and behavioral assessments in two groups after one year. The monthly expenses on phenobarbital, phenytoin and carbamazepine for a $20 \mathrm{~kg}$ child are 1.5,1 and 1.5 USD, respectively.

In sum, drug treatment costs can be lower by prescribing some of the forgotten drugs from the core list of the WHO Model List of Essential Medicines for Children. Cotrimoxazole and chloramphenicol as antimicrobials, chloroquine for malaria, adrenaline, and theophylline for asthma, and phenobarbital as anti-epileptic drug merit place among present-day prescriptions for the common pediatric illnesses.

\section{REFERENCES}

[1] Kesselheim AS, Avorn J, Sarpatwari A. The high cost of prescription drugs in the United States: Origins and prospects for reform. JAMA. 2016; 316(8): 858-871.

[2] Medical costs push millions of people into poverty across the globe. [Online] Available from: https://www.who.int/mediacentre/news/releases/previous/en.

[3] Medical debt pushed 55 million Indians into poverty in one year. [Online] Available from: https://thelogicalindian.com/storyfeed/awareness/medical-debt-pushed-million-indianspoverty/?infinitescroll $=1$.

[4] WHO model list of essential medicines for children? Fifth list. [Online] 2015. Available from: https://www.who.int/medicines/publications/essentialmedicines/en/.

[5] The management of acute respiratory infection in children. World Health Organization, Geneva, 1995

[6] Winberg GA. Occult bacteremia. [Online] Available from: https://www.msdmanuals.com/en-gb/home/children-s-healthissues/bacterial-infections-in-infants-and-children/occult-bacteremia.

[7] Leblebicioglu H, Ozaras R, Sunbul M. Role of co-trimoxazole for urinary tract infections in developing countries. Lancet Infectieous Disease. 2015; 15: 764-65.

[8] Christie CDC, Heikens GT, Gold MHN. Coagulase-negative staphylococcal bacteremia among severely malnourished Jamaican children. The Pediatric Infectious Disease Journal. 1992; 11: 1030-36.

[9] Bishara J, Pitlik S, Samra Z, Levy I, Paul M, Leibovici L. Cotrimoxazole-sensitive, methicillin-resistant staphylococcus aureus, Israel, 1988-1997. Emerging Infectious Diseases. 2003; 9(9): 11681169.

[10] Daga SR, Patil VB, Verma B. Syndromic management of prolonged fever: a cost-effective approach. Tropical Doctor. 2005; 35: 31-34.

[11] Revised WHO classification and treatment of childhood pneumonia at health facilities. World Health Organization, 2014.

[12] Eliakim-Raz N, Lador A, Leibovici-Weissman Y, Elbaz M, Paul M, Leibovici L. Efficacy and safety of chloramphenicol: joining the revival of old antibiotics? Systematic review and meta-analysis of randomized controlled trials. Journal of Antimicrobial Chemotherapy. 2015; 70: 979-996.

[13] Ndu IK, Asinobi IN, Ikwochi U, Amadi OF. Discharge against medical advice (DAMA) among the paediatric age group in Enugu State University Teaching Hospital Parklane, Enugu. Journal of Experimental Research. 2016; 4(1).

[14] Chloramphenicol. In Meyler's Side Effects of Drugs (Sixteenth Edition), 2016.

[15] Guidelines for treatment of malaria, 3rd edition, World Health Organization 2015

[16] Malaria-World Health Organization. [Online] Available from: http://www.who.int/malaria/publications/world-malariareport20156/en/.

[17] Chloroquine and hydroxychloroquine. In Meyler's Side Effects of Drugs (Sixteenth Edition), 2016.

[18] WHO /Fact Sheet: World Malaria Report 2015.

[19] Laufer MK, Thesing PC, Eddington ND, Masonga R, Dzinjalamala FK, Takala SL, et al. Return of chloroquine antimalarial efficacy in Malawi. New England Journal of Medicine. 2006; 355: 1959-66.

[20] Indinnimeo L, Chiappini E, Miraglia del Giudice M. Guideline on management of the acute asthma attack in children by the Italian Society of Pediatrics. Italian Society of Pediatrics. 2018; 44(1): 46.
[21] Becker AB, Nelson NA, Simons FE. Inhaled salbutamol (albuterol) vs. injected epinephrine in the treatment of acute asthma in children. Journal of Pediatrics. 1983; 102: 465-469.

[22] Singhi S, Mathew JL, Torzillo P. What is the role of subcutaneous adrenaline in the management of acute asthma? [Online] 2006. Available from: www.ichrc.org.

[23] Gong W, Jiang J, Fan H. The role of hypodermic injection with a low dose of adrenaline in the emergency treatment of severe asthma. Pharmaceutical Bioprocessing. 2018; 6(3).

[24] Beasley R, Roche WR, Roberts JA, Holgate ST. Cellular events in bronchi in mild asthma and after bronchial provocation American Review of Respiratory Disease. 1989; 139: 806-817.

[25] Giraud V, N. Roche N. Misuse of corticosteroid metered-dose inhaler is associated with decreased asthma stability. European Respiratory Journal. 2002; 19: 246-251.

[26] Lee-Wong M, Mayo PH. Results of a programme to improve house staff use of metered-dose inhalers and spacers. Postgraduate Medical Journal. 2003; 79: 221-5.

[27] Sullivan P, Beziers, Zaffar Z, Page C, Jeffery P, Costello J. Antiinflammatory effects of low dose oral theophylline in atopic asthma. Lancet. 1994, 343: 1006-08.

[28] Hendele S, Weinberger M, Szeflers P, Ellis E. Safety and efficacy of theophylline in children with asthma. Journal of Pediatrics. 1992; 120: 177-83.

[29] Daga S, Verma B, Mhapankar A, Kulkarni S, Kamble P. Theophylline in chronic asthma: A "before and after" study. The Internet Journal of Pediatrics and Neonatology. 2007; 8(1).

[30] The management of acute respiratory infection in children. World Health Organization, Geneva, 1995.

[31] Kale R. The treatment gaps. Epilepsia. 2002; 43(suppl 6): S31-3.

[32] Kale R, Perucca E. Revisiting phenobarbital for epilepsy. BMJ. 2004; 329: 1199-200.

[33] Banu SH, Jahan M, Koli UK, Ferdousi S, Khan NZ, Neville B. Sideeffects of phenobarbital and carbamazepine in childhood epilepsy: a randomized controlled trial. BMJ. 2007; 334(7605). 